\title{
CHARACTERIZATION OF RINGS USING QUASIPROJECTIVE MODULES. III
}

\author{
JONATHAN S. GOLAN
}

\begin{abstract}
A ring $R$ is regular [completely reducible] if and only if the character module of every left $R$-module is quasiinjective [quasiprojective]. Submodules of quasiprojective left $R$-modules over a left perfect ring $R$ are quasiprojective if and only if singular left $R$-modules are injective. A splitting theorem for right perfect rings over which submodules of quasiprojective left $R$-modules are quasiprojective is also proven. These results continue the author's previous work ([5] and [6]).
\end{abstract}

A left $R$-module $M$ is quasiprojective if and only if, for every epimorphism $\alpha::_{R} M \rightarrow_{R} N, \operatorname{Hom}_{R}(M, N)=\operatorname{Hom}_{R}(M, M) \alpha$. Dually, $M$ is quasi-injective if and only if, for every monomorphism $\beta:{ }_{R} N \rightarrow_{R} M$, $\operatorname{Hom}_{R}(N, M)=\beta \operatorname{Hom}_{R}(M, M)$. In [5] and [6] we showed how quasiprojective modules can be used to characterize the rings over which they are defined. Here we will prove additional ring characterizations using quasiprojective and quasi-injective modules.

In what follows $R$ will always denote an associative ring with identity and modules and morphisms will always be taken from the category $R$-mod [resp. mod- $R$ ] of left [resp. right] unitary $R$-modules. Morphisms will always be written acting on the side opposite ring multiplication.

The results presented here are contained in the author's doctoral dissertation, written at the Hebrew University of Jerusalem under the kind direction of Professor S. A. Amitsur. The author also wishes to thank Professor Joseph Rotman with whom he had many stimulating and fruitful conversations.

1. Character modules. Let $G$ be an injective cogenerator over the ring $Z$ of integers and let $\chi=\operatorname{Hom}_{z}(, G)$ be the $G$-character functor. Then $\chi$ can be considered as a faithful exact contravariant functor $R$-mod $\rightarrow$ mod- $R$ [or mod- $R \rightarrow R$-mod] which commutes with finite direct sums. Furthermore, for any $R$-module $M$ we have a canonical

Received by the editors February 1, 1971.

AMS 1969 subject classifications. Primary 1650.

Key words and phrases. Quasiprojective module, character module, completely reducible ring, regular ring, hereditary ring, semihereditary ring, perfect ring. 
embedding $M \rightarrow \chi^{2}(M)$. The module $\chi(M)$ is called the character module of $M$ (with respect to $G$ ). The major results of this section are:

THEOREM A. A ring $R$ is regular (in the sense of von Neumann) if and only if the character module of every left $R$-module is quasi-injective.

THEOREM B. The following are equivalent for a ring $R$ :

(1) $R$ is completely reducible.

(2) $\chi(M)$ is quasiprojective for every right [left] $R$-module $M$.

(3) $\chi^{2}(M)$ is quasiprojective for every left [right] $R$-module $M$.

We will adopt the following notation from [11]: If $M$ is a left $R$-module, then

$\pi^{-1}(M)$ will denote the class of all epimorphisms $\alpha:{ }_{R} U \rightarrow_{R} V$ such that Hom $\left(\operatorname{id}_{M}, \alpha\right): \operatorname{Hom}_{R}(M, U) \rightarrow \operatorname{Hom}_{R}(M, V)$ is an epimorphism.

$\iota^{-1}(M)$ will denote the class of all monomorphisms $\beta:_{R} U \rightarrow_{R} V$ such that $\operatorname{Hom}\left(\beta, \mathrm{id}_{M}\right): \operatorname{Hom}_{R}(V, M) \rightarrow \operatorname{Hom}_{R}(U, M)$ is an epimorphism.

$\tau^{-1}(M)$ will denote the class of all monomorphisms $\gamma: U_{R} \rightarrow V_{R}$ such that $\gamma \otimes \mathrm{id}_{M}: U \otimes_{R} M \rightarrow V \otimes_{R} M$ is a monomorphism.

Let $M$ be a left $R$-module and $U$ a right $R$-module. As a special case of the homological duality formulae [3, Propositions II,5.2 and II,5.2'] we have the canonical isomorphisms

$$
\operatorname{Hom}_{R}(U, \chi(M)) \cong \chi\left(U \otimes_{R} M\right) \cong \operatorname{Hom}_{R}(M, \chi(U))
$$

from which we can immediately infer:

(1.1) Lemma. If $M$ is a left $R$-module and $\alpha: U_{R} \rightarrow V_{R}$ is a homomorphism, then the following are equivalent:

(i) $\alpha \in \tau^{-1}(M)$.

(ii) $\alpha \in \iota^{-1}(\chi(M))$.

(iii) $\chi(\alpha) \in \pi^{-1}(M)$.

(See [11, Proposition 3.2].)

If $M$ is a finitely-presented left $R$-module and $N$ is an arbitrary left $R$-module, then as a special case of the isomorphism given in $[1$, p. 63, Exercise 14] we have a canonical isomorphism

$$
\chi(N) \otimes_{R} M \cong \chi\left(\operatorname{Hom}_{R} \cdot(M, N)\right) .
$$

From this and Lemma 1.1 we then have:

(1.2) LemMA. Let $M$ be a finitely-presented left $R$-module and let $\beta:{ }_{R} U \rightarrow_{R} V$ be an epimorphism. Then the following are equivalent :
(i) $\beta \in \pi^{-1}(M)$.
(ii) $\chi(\beta) \in \iota^{-1}(\chi(M))$.
(iii) $\chi^{2}(\beta) \in \pi^{-1}(M)$. 
(1.3) Corollary. If $M$ is a finitely-presented left $R$-module and $\chi(M)$ is quasi-injective then $M$ is quasiprojective.

Proof. If $\alpha:{ }_{R} M \rightarrow_{R} V$ is an epimorphism then $\chi(\alpha): \chi(V)_{R} \rightarrow \chi(M)_{R}$ is a monomorphism and so, by the quasi-injectivity of $\chi(M)$, belongs to $\iota^{-1}(\chi(M))$. By Lemma 1.2, $\alpha$ then belongs to $\pi^{-1}(M)$, proving $M$ quasiprojective.

We are now in a position to prove our main theorems.

Proof of Theorem A. If $R$ is regular then every left $R$-module is flat. Lambek [9, p. 131] has shown that a left $R$-module $M$ is flat if and only if $\chi(M)$ is injective so, in particular, $\chi(M)$ is clearly quasi-injective.

Conversely assume that $R$ is not regular. Then there exists a left $R$ module $M$ which is not flat; that is to say, there exist right $R$-modules $U_{R}$ and $V_{R}$ together with a monomorphism $\lambda: U_{R} \rightarrow V_{R}$ such that $\lambda \otimes \operatorname{id}_{M}: U \otimes_{R} M \rightarrow V \otimes_{R} M$ is not a monomorphism. Let ${ }_{R} T=$ $\chi(V) \oplus M$. Then $\chi(T) \cong \chi^{2}(V) \oplus \chi(M)$ and we have the canonical embeddings $\rho: V \rightarrow \chi^{2}(V) \rightarrow \chi^{2}(V) \oplus \chi(M) \cong \chi(T)$ and $\sigma: M \rightarrow T$.

If $\quad 0 \neq \sum_{i=1}^{n}\left(u_{i} \otimes m_{i}\right) \in \operatorname{ker}\left(\lambda \otimes \operatorname{id}_{M}\right)$ then $0 \neq \sum_{i=1}^{n}\left(u_{i} \otimes m_{i} \sigma\right) \in U \otimes_{R} T$ and

$$
\left[\sum_{i=1}^{n}\left(u_{i} \otimes m_{i} \sigma\right)\right]\left(\lambda \rho \otimes \mathrm{id}_{T}\right)=\left[\sum_{i=1}^{n}\left(u_{i} \otimes m_{i}\right)\right]\left(\lambda \otimes \mathrm{id}_{M}\right)(\rho \otimes \sigma)=0,
$$

proving that $\lambda \rho \otimes \mathrm{id}_{T}: U \otimes_{R} T \rightarrow \chi(T) \otimes_{R} T$ is not a monomorphism, i.e., $\lambda \rho \notin \tau^{-1}(T)$. By Lemma 1.1, this means that $\lambda \rho \notin \iota^{-1}(\chi(T))$, proving that $\chi(T)$ is not quasi-injective.

ProOF OF THEOREM B. The implications $(1) \Rightarrow(2) \Rightarrow(3)$ are trivial. Therefore assume (3). If $N$ is an injective left $R$-module then $N$ can be canonically embedded in $\chi^{2}(N)$ and so is a direct summand of $\chi^{2}(N)$. Since $\chi^{2}(N)$ is quasiprojective, so is $N$ and so we have that every injective left $R$-module is quasiprojective, which proves that $R$ is a quasifrobenius ring [2, Corollary 2.3]. In particular, $R$ is left noetherian.

Every finitely-generated left $R$-module over a left noetherian ring is finitely-presented $[1, \mathrm{p}$. 36]. Since $R$ is completely reducible iff every finitely-generated left $R$-module is quasiprojective (the proof is the same as that of Theorem 1.3 of [5]) it suffices by Corollary 1.3 to show that $\chi(M)$ is quasi-injective for every finitely-generated left $R$-module $M$. Let $\alpha: U_{R} \rightarrow \chi(M)_{R}$ be a monomorphism. Then $\chi^{3}(\alpha): \chi^{4}(M) \rightarrow \chi^{3}(U)$ is an epimorphism. But $\chi^{4}(M) \oplus \chi^{3}(U) \cong \chi^{2}\left(\chi^{2}(M) \oplus \chi(U)\right)$ which is quasiprojective. Since a sufficient condition for an epimorphism ${ }_{R} X \rightarrow_{R} Y$ to be a retraction is that $X \oplus Y$ be quasiprojective [6, Lemma 2.1], it follows that $\chi^{3}(\alpha)$ is a retraction and hence surely belongs to $\pi^{-1}(M)$. By Lemma 
1.2, $\chi(\alpha)$ therefore belongs to $\pi^{-1}(M)$ and so Lemma 1.1 implies that $\alpha$ belongs to $\iota^{-1}(\chi(M))$. This proves that $\chi(M)$ is quasi-injective.

A submodule $N$ of a left $R$-module $M$ is pure if and only if, for every right $R$-module $T$, the canonical imbedding $N \rightarrow M$ belongs to $\tau^{-1}(T)$. An $R$-module $U$ is pure-injective if and only if, for every pure submodule $N$ of an $R$-module $M$, the embedding $N \rightarrow M$ belongs to $\iota^{-1}(U)$.

If as our injective cogenerator $G$ we take as a special case $R / Z$, where $\boldsymbol{R}$ is the field of reals, then $\chi^{2}(M)$ is a compact $R$-module and $M$ is a pure submodule of $\chi^{2}(M)$. Moreover, a module $U$ is pure-injective if and only if it is a summand of $\chi^{2}(U)$ [12, pp. 706-708]. We then get the following corollary to Theorem B, generalizing a result of Griffith [7]:

(1.4) Corollary. A ring $R$ is completely reducible if and only if every pure-injective left $R$-module is quasiprojective.

2. Heredity with respect to quasiprojectives. In [6] we looked briefly at rings $R$ satisfying the condition that every [finitely-generated] submodule of a quasiprojective left $R$-module be quasiprojective. Such rings we will call left [semi-] HQ-rings. This condition is stronger than left hereditary and in fact we have shown [6, Theorem 5.1] that every factor ring of a left [semi-] $H Q$-ring is left [semi-] hereditary. If $R$ is left perfect then the converse also holds. We will now characterize these rings in more detail; our main results are:

THEOREM C. A left perfect ring $R$ is a left HQ-ring if and only if every singular left $R$-module is injective.

THEOREM D. If $R$ is a right perfect left semi-HQ ring then $R$ admits a splitting $R=S \oplus J(R)$ over $Z$, where $S$ is a completely reducible subring of $R$ containing 1 .

A two-sided ideal $I$ of a ring $R$ is left $T$-nilpotent if and only if, for every sequence $\left\langle a_{i}\right\rangle$ of elements of $I$, there exists an integer $n$ such that $a_{1} \cdot \ldots \cdot a_{n}=0$. Ideals satisfying this condition were first studied by Levitzki [10]. The following lemma is known:

(2.1) Lemma. Let $I$ be a left T-nilpotent two-sided ideal of a ring $R$. Then for every left $R$-module $M, I M$ is small in $M$.

Proof. ${ }_{R} U$ is small in ${ }_{R} V$ if and only if $U+{ }_{R} W=V$ implies $W=V$. Assume $I M$ is not small in $M$. We will arrive at a contradiction by constructing a sequence $\left\langle a_{i}\right\rangle$ of elements of $I$ such that $a_{1} \cdot \ldots \cdot a_{n} \neq 0$ for all $n$.

Since $I M$ is not small in $M$ there exists a proper submodule $N$ of $M$ with $I M+N=M$. In particular there exists $a_{1} \in I$ and $m_{1} \in M$ such 
that $a_{1} m_{1} \notin N$ (and hence surely $a_{1} m_{1} \neq 0$ ). Now assume inductively that there exists $a_{1}, \cdots, a_{n} \in I$ and $m_{n} \in M$ such that $a_{1} \cdot \ldots \cdot a_{n} m_{n} \notin N$. Then $m_{n}=y+\sum_{j=1}^{k} b_{j} x_{j}$ for $y \in N, \quad b_{j} \in I$, and $x_{j} \in M$. Since $a_{1} \cdot \ldots \cdot a_{n} m_{n} \notin N$ there exists some $j$ such that $a_{1} \cdot \ldots \cdot a_{n} b_{j} x_{j} \notin N$. Take $a_{n+1}=b_{j}$ and $m_{n+1}=x_{j}$.

(2.2) Proposition. Let $R$ be a left $H Q$-ring and let $H$ be a left $T$ nilpotent two-sided ideal of $R$. Then $H^{2}=0$. In particular, if $R$ is left perfect then $J(R)^{2}=0$.

Proof. Since $H$ is left $T$-nilpotent, $H=H R$ is small in $R$ and so $H$ is contained in the Jacobson radical $J(R)$ of $R$ (which equals the union of all small left ideals of $R$ ). Let $S=R / H^{2}$. Since $R$ is a left $H Q$-ring, $R / H^{2}$ is left hereditary and so, in particular, $J(R) / H^{2}$ is a projective left $S$-module since it is a left ideal of $S$. Let ${ }_{S} U=J(R) / H^{3}$ and ${ }_{S} V=H^{2} / H^{3}$. Then $U / V$ is $S$-isomorphic to $J(R) / H^{2}$ and so is projective over $S$. But $V \subseteq H U$ and so is small in $U$. Thus by the splitting of the exact sequence $0 \rightarrow V \rightarrow U \rightarrow U / V \rightarrow 0$ over $S$ we see that $V$ is a small direct summand of $U$ and so $V=0$. This proves that $H^{2}=H^{3}$. But by Lemma $2.1, H^{3}$ is small in $H^{2}$ and so $H^{2}=0$.

The second part of the theorem comes from the well-known fact that the Jacobson radical of a left perfect ring is left $T$-nilpotent.

(2.3) Corollary. Let $R$ be a left hereditary left perfect ring. Then the following are equivalent:

(1) $R$ is a left $H Q$-ring.

(2) $J(R)^{2}=0$.

Proof. $\quad(1) \Rightarrow(2)$ follows directly from Proposition 2.2.

$(2) \Rightarrow(1)$ : Harada has shown that if $R$ is left hereditary and semiprimary and if $k$ is the index of nilpotency of $J(R)$ then for any two-sided ideal $I$ of $R$, l.gl. $\operatorname{dim}(R / I) \leqq k-1$ [8, Theorem 6]. In particular, (2) implies that $R / I$ is left hereditary for every two-sided ideal $I$ of $R$ and so $R$ is a left $H Q$-ring since it is left perfect.

Recall that a submodule $N$ of a module $M$ is large if and only if it has a nonzero intersection with any nonzero submodule of $M$ and that the singular submodule $Z(M)$ of a left $R$-module $M$ is given by $Z(M)=$ $\{m \in M \mid(0: m)$ is a large left ideal of $R\}$. An $R$-module $M$ is singular if and only if $Z(M)=M$ and nonsingular if and only if $Z(M)=0$. Both the class of singular $R$-modules and the class of nonsingular $R$-modules are closed under taking submodules.

Proof of Theorem C. Let $R$ be a left perfect ring and assume that $R$ is a left $H Q$-ring as well. Let $M$ be a singular left $R$-module. To show that 
$M$ is injective it suffices to show that every diagram of the form

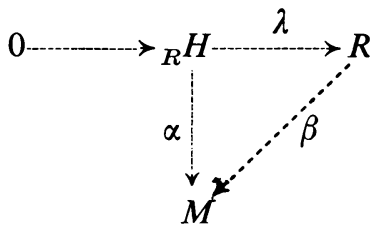

can be completed commutatively, where $H$ is a large left ideal of $R, \lambda$ is the canonical embedding, and $\alpha$ is an arbitrary nonzero homomorphism [9, p. 93].

Let $K=\operatorname{ker}(\alpha)$ and suppose that $K$ is not large in $R$. Then there exists a nonzero left ideal $I$ of $R$ such that $K \cap I=0$. Since $H$ is large in $R$, $I^{\prime}=H \cap I$ is nonzero and $I^{\prime} \cap K=0$. Thus the restriction of $\alpha$ to $I^{\prime}$ is a monomorphism and so $I^{\prime} \cong I^{\prime} \alpha$ which is a submodule of a singular module and so is singular. On the other hand, $R$ is in particular left hereditary and so is nonsingular. Therefore $I^{\prime}$, a submodule of $R$, is also nonsingular, yielding a contradiction which proves that $K$ is large in $R$.

By Proposition $2.2, J(R)^{2}=0$ and so $J(R)$ is a left $R / J(R)$-module and so is completely reducible for, by the left perfectness of $R, R / J(R)$ is a completely reducible ring. Therefore $J(R) \subseteq \operatorname{soc}\left({ }_{R} R\right)$. But the socle of a left $R$-module is precisely the intersection of all of its large submodules and so, in particular, $J(R) \subseteq K$. Therefore $R / K$ is a left $R / J(R)$-module and thus is completely reducible.

The map $\alpha$ induces a monomorphism $\bar{\alpha}: H / K \rightarrow M$. Since $R / K$ is a completely reducible $R$-module, $H / K$ is a direct summand of $R / K$ and so we have a canonical projection $\pi: R / K \rightarrow H / K$. Finally, let $v: R \rightarrow R / K$ be the canonical epimorphism. Then $\nu \pi \bar{\alpha}$ is an $R$-homomorphism $R \rightarrow M$ and for every $h \in H, h \nu \pi \bar{\alpha}=(h+K) \pi \bar{\alpha}=(h+K) \bar{\alpha}=h \alpha$ so $\beta=\nu \pi \bar{\alpha}$ is the homomorphism we seek.

Conversely, assume that $R$ is a left perfect ring over which every singular module is injective. Let $I$ be a two-sided ideal of $R$ and let $S=R / I$. For $M$ a left $S$-module let ${ }_{S} \hat{M}$ be the injective hull of $M$ over $S$. Then ${ }_{R} M$ is large in ${ }_{R} \hat{M}$ and so, as is easily seen, $\hat{M} / M$ is a singular left $R$-module. Therefore $\hat{M} / M$ is injective over $R$ and hence injective over $S$. Since the sequence $0 \rightarrow M \rightarrow \hat{M} \rightarrow \hat{M} / M \rightarrow 0$ is exact over $S$, inj $\operatorname{dim}\left({ }_{S} M\right) \leqq 1$. Since this is true for any left $S$-module $M, S$ is left hereditary [3, p. 112] and so $R$ is a left $H Q$-ring by Theorem 5.1 of [6].

Note that the argument in the above proof is based on the techniques of [4].

(2.4) Corollary. If $R$ is a left perfect left $Q H$-ring then $Z(M)$ is a direct summand of $M$ for every left $R$-module $M$. 
We now turn to look at right perfect left semi- $H Q$-rings, and use techniques based on those of Zaks [13] to obtain a classical splitting theorem (Theorem D).

(2.5) Lemma. Let $R$ be a right perfect left semi-HQ-ring. If $e$ and $f$ are indecomposable idempotents of $R$ with $e R f$ and fRe nonzero, then $R e \cong R f$ and in fact this isomorphism is given by right multiplication by any nonzero element of $e R f$ or fRe.

Proof. Let $a, b$ be elements of $R$ such that eaf and fbe are nonzero. Define $\alpha: R e \rightarrow R f$ by $r e \mapsto r e a f$. Then $R e \oplus R e a f$ is a finitely-generated submodule of $R \oplus R$ and so is quasiprojective since $R$ is a left semi-HQring. Since $\alpha$ is an epimorphism of $R e$ onto Reaf and $R e$ is projective, it follows from Lemma 1.1 of [5] that Reaf is projective and that $\alpha$ is a retraction. Since $R e$ is indecomposable, $\alpha$ is then a monomorphism.

Similarly the homomorphism $\beta: R f \rightarrow R e$ given by $r f \mapsto r f b e$ is in fact a monomorphism. We are therefore done if we can show that $R \boldsymbol{e}=$ $\operatorname{im}(\alpha \beta)=$ Reafbe for then $\beta$ would be an epimorphism and hence an isomorphism.

But we have a descending sequence $\operatorname{Re} \supseteq \operatorname{Re} \alpha \beta \supseteq \operatorname{Re}(\alpha \beta)^{2} \supseteq \cdots$ of principal left ideals of $R$ which must terminate since $R$ is right perfect and therefore satisfies the descending chain condition on principal left ideals. Therefore there exists an integer $n$ such that $\operatorname{Re}(\alpha \beta)^{n}=\operatorname{Re}(\alpha \beta)^{n+1}$. In particular, $e(\alpha \beta)^{n}=r e(\alpha \beta)^{n+1}$ for some $r \in R$ and so $e-r e \alpha \beta \in$ $\operatorname{ker}(\alpha \beta)^{n}$. But $\alpha$ and $\beta$ are both monomorphisms so this kernel equals 0 . Thus $e=r e \alpha \beta$ and so $R e=\operatorname{Re} \alpha \beta$.

(2.6) Corollary. If $R$ is a right perfect left semi-HQ-ring and $e$ is an indecomposable idempotent of $R$ then $e R e$ is a division ring.

Proof of Theorem D. Let $R$ be a right perfect left semi- $H Q$-ring. Then there exists a set $\left\{e_{1}, \cdots, e_{n}\right\}$ of indecomposable orthogonal idempotents of $R$ such that $R=\oplus_{i=1}^{n} R e_{i}$ and each $R e_{i} / J(R) e_{i}$ is a simple left $R$-module. Furthermore, we have the $Z$-decomposition

$$
R=\oplus_{i=1}^{n} \bigoplus_{j=1}^{n} e_{i} R e_{j} \text {. }
$$

Fix some index $k, 1 \leqq k \leqq n$, and let $S_{k}=\sum e_{i} R e_{j}$ where the sum ranges over all indices $i, j$ such that $R e_{i} \cong R e_{j} \cong R e_{k}$. Then $S_{k}$ is a subring of $R$ which is isomorphic to the full matrix ring $\left(e_{k} R e_{k}\right)_{t}$, where $t$ is the number of different indices $i$ in the above sum. Since $e_{k} R e_{k}$ is a division ring by Corollary $2.6, S_{k}$ is a simple ring.

Now let $S=\sum_{k=1}^{n} S_{k}$. Then this is a subring of $R$ and, since each $S_{k}$ is simple, $S$ is completely reducible and contains $1=e_{1}+\cdots+e_{n}$. Let $T=\sum e_{i} R e_{j}$ where the sum ranges over all indices $i, j$ such that 
$R e_{i}$ and $R e_{j}$ are not isomorphic. We will be done if we can show that $T=J(R)$. Clearly $T \subseteq J(R)$ since we have the chain of implications $e_{i} a e_{j} \notin J(R) \Rightarrow R e_{i} a e_{j} \notin J(R) e_{j} \Rightarrow R e_{i} a e_{j}=R e_{j} \Rightarrow$ the homomorphism $R e_{i} \rightarrow R e_{j}$ given by $r e_{i} \mapsto r e_{i} a e_{j}$ is an isomorphism $\Rightarrow e_{i} a e_{j} \notin T$. Since $S$ is completely reducible and $R=S+T$, to show that $T=J(R)$ it therefore suffices to show that $T$ is a two-sided ideal of $R . T$ is clearly closed under addition. Let $e_{i} a e_{j} \in T$ and consider $0 \neq y=e_{k} b e_{i} a e_{j}$. If $R e_{k}$ is not isomorphic to $R e_{j}$ then $y \in e_{k} R e_{j} \subseteq T$. Hence assume that $R e_{k} \cong$ $R e_{j}$. If $\beta: R e_{k} \rightarrow R e_{i}$ and $\alpha: R e_{i} \rightarrow R e_{j}$ are the $R$-homomorphisms respectively given by $r e_{k} \mapsto r e_{k} b e_{i}$ and $r e_{i} \mapsto r e_{i} a e_{j}$ then by the same reasoning as in Lemma 2.5 each of these maps is a monomorphism. But $\beta \alpha$ is just the map given by right multiplication by $y$ and so, by Lemma 2.5 , is an isomorphism between $R e_{k}$ and $R e_{j}$. Therefore $\alpha$ must also be an epimorphism and so is an isomorphism between $R e_{i}$ and $R e_{j}$, a contradiction. Hence $y=0$ for $R e_{k} \cong R e_{j}$ and so $R T \subseteq T$. A similar proof shows $T R \subseteq T$.

\section{REFERENCES}

1. N. Bourbaki, Algèbre commutative. Chaps. 1, 2, Actualités Sci. Indust., no. 1290, Hermann, Paris, 1961. MR 36 \#146.

2. K. A. Byrd, Some characterizations of uniserial rings, Math. Ann. 186 (1970), 163-170.

3. H. Cartan and S. Eilenberg, Homological algebra, Princeton Univ. Press, Princeton, N.J., 1956. MR 17, 1040.

4. V. C. Cateforis and F. L. Sandomierski, The singular submodule splits off, J. Algebra 10 (1968), 149-165. MR 39 \#2805.

5. J. S. Golan, Characterization of rings using quasiprojective modules, Israel J. Math. 8 (1970), 34-38.

6. - Characterization of rings using quasiprojective modules. II, Proc. Amer. Math. Soc. 28 (1971), 337-343.

7. P. Griffith, A note on a theorem of Hill, Pacific J. Math. 29 (1969), 279-284. MR 39 \#6919.

8. M. Harada, Hereditary semi-primary rings and tri-angular matrix rings, Nagoya Math. J. 27 (1966), 463-484. MR 34 \#4300.

9. J. Lambek, Lectures on rings and modules, Blaisdell, Waltham, Mass., 1966. MR 34 \#5857.

10. J. Levitzki, Contributions to the theory of nilrings, Riveon Lematematika 7 (1954), 50-70. (Hebrew) MR 15, 677.

11. Bo. T. Stenström, Pure submodules, Ark. Mat. 7 (1967), 159-171. MR 36 \#6473.

12. R. B. Warfield, Purity and algebraic compactness for modules, Pacific J. Math. 28 (1969), 699-719. MR 39 \#4212.

13. A. Zaks, Semiprimary rings of generalized triangular type, J. Algebra 9 (1968), 54-78. MR 36 \#6455.

Institute of Mathematics, The Hebrew University of Jerusalem, Jerusalem, ISRAEL

Current address: Department of Mathematics, University of Florida, Gainesville, Florida 32601 